Article

\title{
Pyrene Coating Transition Metal Disulfides as Protection from Photooxidation and Environmental Aging
}

\author{
Ruben Canton-Vitoria ${ }^{1,+}+{ }^{\circ}$, Yuman Sayed-Ahmad-Baraza ${ }^{2,+}+\mathbb{C}$, Bernard Humbert $^{2}$, \\ Raul Arenal ${ }^{3,4,5, * \mathbb{C}}$, Christopher P. Ewels ${ }^{2, *}$ and Nikos Tagmatarchis ${ }^{1, *(\mathbb{D})}$ \\ 1 Theoretical and Physical Chemistry Institute, National Hellenic Research Foundation, \\ 48 Vassileos Constantinou Avenue, 11635 Athens, Greece; canton@eie.gr \\ 2 Institut des Materiaux Jean Rouxel (IMN), UMR6502 CNRS, Universite de Nantes, 2 Rue de la Houssiniere, \\ BP32229, 44322 Nantes, France; yusabar@gmail.com (Y.S.-A.-B.); bernard.humbert@cnrs-imn.fr (B.H.) \\ 3 Laboratorio de Microscopias Avanzadas, Instituto de Nanociencia de Aragon, Universidad de Zaragoza, \\ 50018 Zaragoza, Spain \\ 4 ARAID Foundation, 50018 Zaragoza, Spain \\ 5 Instituto de Ciencias de Materiales de Aragon, CSIC-U. Zaragoza, 50009 Zaragoza, Spain \\ * Correspondence: arenal@unizar.es (R.A.); chris.ewels@cnrs-imn.fr (C.P.E.); tagmatar@eie.gr (N.T.) \\ $\dagger$ These authors contributed equally to this work.
}

Received: 29 January 2020; Accepted: 14 February 2020; Published: 19 February 2020

check for updates

\begin{abstract}
Environmental degradation of transition metal disulfides (TMDs) is a key stumbling block in a range of applications. We show that a simple one-pot non-covalent pyrene coating process protects TMDs from both photoinduced oxidation and environmental aging. Pyrene is immobilized non-covalently on the basal plane of exfoliated $\mathrm{MoS}_{2}$ and $\mathrm{WS}_{2}$. The optical properties of TMD/pyrene are assessed via electronic absorption and fluorescence emission spectroscopy. High-resolution scanning transmission electron microscopy coupled with electron energy loss spectroscopy confirms extensive pyrene surface coverage, with density functional theory calculations suggesting a strongly bound stable parallel-stacked pyrene coverage of $\sim 2-3$ layers on the TMD surfaces. Raman spectroscopy of exfoliated TMDs while irradiating at $0.9 \mathrm{~mW} / 4 \mu \mathrm{m}^{2}$ under ambient conditions shows new and strong Raman bands due to oxidized states of Mo and W. Yet remarkably, under the same exposure conditions TMD/pyrene remain unperturbed. The current findings demonstrate that pyrene physisorbed on $\mathrm{MoS}_{2}$ and $\mathrm{WS}_{2}$ acts as an environmental barrier, preventing oxidative surface reactions in the TMDs catalyzed by moisture, air, and assisted by laser irradiation. Raman spectroscopy confirms that the hybrid materials stored under ambient conditions for two years remained structurally unaltered, corroborating the beneficial role of pyrene for not only hindering oxidation but also inhibiting aging.
\end{abstract}

Keywords: $\mathrm{MoS}_{2} ; \mathrm{WS}_{2} ; 2 \mathrm{D}$ materials; exfoliation; functionalization; pyrene; oxidation; aging; protection; DFT calculations

\section{Introduction}

Transition metal disulfides (TMDs) consist of covalently bound chalcogen-metal-chalcogen layers interacting weakly via van der Waals forces. Wet processing of bulk TMDs is currently the most efficient top-down approach for yielding stable colloidal dispersions of high-quality exfoliated layers [1-4] enabling easier handling and manipulation. In TMDs, the layer surface is terminated by chalcogen electron lone pairs and thus no dangling bonds are present. Nevertheless, a handicap of the unique structure of monolayer TMDs is the direct exposure of all atoms to the environment, which can 
induce significant structural modifications, and affect the novel electronic, optical, and mechanical properties [5,6]. Edges and defect sites are susceptible to oxidation, under atmospheric conditions and/or moisture when exposed to light illumination. This forms oxides which can significantly degrade the performance in energy and tribology related applications [7]. Even 'defect-free' TMDs oxidize at the basal plane when exposed to atomic oxygen found in low earth orbit environment [8-11], which can severely impact their use as lubricants in space technology. In addition, the presence of oxygen on the surface of TMDs is a critical issue in micro- and opto-electronic applications, where high electrical conductivity and carrier mobility is required. Chemical vapor deposition grown monolayer $\mathrm{MoS}_{2}$ and $\mathrm{WS}_{2}$ have been shown to degrade over time even at room temperature, with device currents dropping almost two orders of magnitude after just one month [12]. Even brief ambient exposure ( $<1 \mathrm{~min})$ was shown to increase contact resistance by over an order of magnitude and decrease intrinsic mobility in $\mathrm{MoS}_{2}$ transistors [13].

Different strategies have been adopted to minimize or eliminate these oxidation processes in TMDs, typically through the use of capping layers. These include encapsulation with other monolayer materials such as h-BN $[14,15]$ and graphene [16], or the use of atomic-layer deposition to cover 2D-materials with an oxide layer [17]. However, the former approaches are not very practical or scalable while the latter is limited to nanosheets deposited on a substrate, and thus they are not adapted to solution-based processing. An alternative is the use of polymer encapsulating layers [12] but this requires thick (10-20 nm) polymer layers and the capping layer is difficult to remove without damaging the TMD. It is clear that a simple, scalable, reversible, and solution-based route to environmental protection of TMDs would be highly desirable.

With the aforementioned points in mind and considering the simplicity and reversibility of non-covalent interactions for associating an electron-rich flat all-sp ${ }^{2}$ hybridized carbon species with exfoliated semiconducting $2 \mathrm{H}-\mathrm{MoS}_{2}$ and $2 \mathrm{H}-\mathrm{WS}_{2}$, we have developed $\mathrm{MoS}_{2} /$ pyrene and $\mathrm{WS}_{2} /$ pyrene ensembles. The $\pi$-S interactions between the pyrene moiety and the TMDs were exploited to build the ensembles, avoiding complex and time-consuming methodologies. Importantly such TMDs can be isolated from ambient oxygen environmental conditions, resulting in high stability even after prolonged laser irradiation exposure.

We first focus on the non-covalent modification of TMDs with an electron-donor moiety and examine the optical characteristics and material properties of the corresponding $\mathrm{MoS}_{2} /$ pyrene and $\mathrm{WS}_{2} /$ pyrene ensembles. Sample morphology is elucidated by high-resolution scanning transmission electron microscopy (HR-STEM) imaging coupled with electron energy loss spectroscopy (EELS) STEM, while complementary electronic absorption and fluorescence emission spectroscopic assays reveal information related with the photophysical properties of the materials and the existence of electronic interactions in the ground and excited states. Density functional theory (DFT) calculations demonstrate the most favorable pyrene stacking and allow estimation of surface coverage. Then, in order to protect the $\mathrm{MoS}_{2}$ and $\mathrm{WS}_{2}$ from environmental attack, coverage of TMDs with pyrene as an oxidation resistant species is scrutinized. This new approach has the advantage of non-covalent modification, leaving intact and undisturbed the surface of TMDs, without affecting negatively their electronic and mechanical properties. Additionally, it protects TMDs from oxidation under ambient conditions (air, moisture, light illumination, and aging), without requiring isolation of the material from the environment, minimizing necessity for high-cost equipment and advanced processes for handling the materials.

\section{Materials and Methods}

General. All chemicals and solvent were purchased from Sigma Aldrich (Merck KGaA and/or its affiliates, Darmstadt, Germany) and used as received unless otherwise stated. Steady-state UV-Vis electronic absorption spectra were recorded on a PerkinElmer (Lambda 19, PerkinElmer Inc. Madrid, Spain) UV-Vis-Near Infrared (NIR) spectrophotometer. Steady-state emission spectra were recorded on a Fluorolog-3 JobinYvon-Spex spectrofluorometer (model GL3-21, Jobin Yvon 
Inc. New York, NCY, USA) with $1 \mathrm{~nm}$ resolution. Pico-second time-resolved fluorescence spectra were measured by the time-correlated-single-photon-counting (TCSPC, PicoQuant GmbH, Berlin, Germany) method on a Nano-Log spectrofluorometer (Horiba JobinYvon, Bensheim, Germany), using a laser diode as an excitation source (NanoLED, $375 \mathrm{~nm}$, Orlando, FL, USA) and a UV-Vis detector TBX-PMT series (250-850 nm, Horiba JobinYvon, Bensheim, Germany) from Horiba JobinYvon. Lifetimes were evaluated with the DAS6 Fluorescence-Decay Analysis Software (Horiba JobinYvon, Bensheim, Germany). Typically, 100 scans were acquired at $2 \mathrm{~cm}^{-1}$ resolution. Micro-Raman scattering measurements were performed at room temperature in the backscattering geometry using a RENISHAW inVia Raman microscope (Renishaw plc, Gloucestershire, UK) equipped with a charge-coupled device (CCD) camera and a Leica microscope. A 2400 lines/mm grating was used for all measurements, providing a spectral resolution of $2 \mathrm{~cm}^{-1}$ and an accuracy of $\pm 5 \mathrm{~cm}^{-1}$. Measurements were taken with $15 \mathrm{~s}$ of exposure times at varying numbers of accumulations. The laser spot was focused on the sample surface using a long working distance $50 \times$ objective (N.A. $=0.35$ ). Raman spectra were collected on numerous spots on the sample and recorded with Peltier cooled CCD camera (Renishaw plc, Gloucestershire, UK). The recorded data were treated with (Renishaw Wire and Origin software (Renishaw plc, Gloucestershire, UK). Thermogravimetric analysis was performed using a TGA Q500 V20.2 Build 27 instrument by TA in a nitrogen (purity > 99.999\%) inert atmosphere. The microwave-assisted reaction was performed in a CEM Discover SP reactor employed in open-batch modality. Spatial-resolved EELS measurements were performed on probe-corrected STEM FEI Titan Low-Base 60-300 operating at $80 \mathrm{keV}$ (fitted with an X-FEG ${ }^{\circledR}$ gun, Eindhoven, Netherlands) and Cs-probe corrector (CESCOR from CEOS GmbH, Heidelberg, Germany). EEL spectra were recorded using the spectrum-imaging (SPIM in 2D or spectrum-line (SPLI) in 1D) mode $[18,19]$ in a Gatan GIF Tridiem ESR 865 spectrometer (Pleasanton, CA, USA). The convergent semi-angle was of $25 \mathrm{mrad}$, the collection semi-angle was $80 \mathrm{mrad}$ and the energy resolution $1.2 \mathrm{eV}$. All electron microscopy and spectroscopy experiments were conducted at low temperature $\left(-170{ }^{\circ} \mathrm{C}\right)$ to avoid damaging of the pyrene entities. The EELS datasets were denoised with the open-source program Hyperspy by using principal component analysis [19].

Exfoliation of $\mathbf{M o S}_{\mathbf{2}}$ and $\mathbf{W S}_{\mathbf{2}}$. Bulk TMDs (150-200 mg) were dispersed in chlorosulfonic acid and sonicated for $2 \mathrm{~h}$ at room temperature. The solution was left under stirring for a month, occasionally sonicated for $30 \mathrm{~s}$. Afterwards cold water was added to the solution under stirring, drop by drop. This was done carefully since the reaction is exothermic and releases gaseous $\mathrm{HCl}$. Next, the mixture was filtrated on a PTFE filter of $0.2 \mu \mathrm{m}$ pore-size and washed with excess methanol and acetone. The solid compound was added to $\mathrm{N}$-methyl pyrrolidone and sonicated for $1 \mathrm{~h}$ (tip sonication at $30-35 \%$ of amplitude $(100 \%$ of $200 \mathrm{~W})$ ). After 3 days the supernatant was taken, filtrated on PTFE filter ( $0.2 \mu \mathrm{m}$ pore-size) and washed with a large amount of methanol, acetone and dichloromethane.

TMD-based nanoensembles $1 \mathbf{a}$ and $\mathbf{1 b}$. A mixture of exfoliated TMDs $(20 \mathrm{mg})$ and pyrene $(10 \mathrm{mg})$ in $\mathrm{N}, \mathrm{N}$-dimethylformamide (DMF) $(10 \mathrm{~mL})$ was bath sonicated for $15 \mathrm{~min}$ followed by stirring at room temperature with small intervals of sonication for a total period of $36 \mathrm{~h}$. Then, the reaction mixture was filtered over a PTFE filter (100 nm pore size) and the solid residue collected onto the filter was washed with dichloromethane to remove excess free pyrene, furnishing $\mathrm{MoS}_{2} /$ pyrene $1 \mathrm{a}$ and $\mathrm{WS}_{2} /$ pyrene $1 \mathrm{~b}$ nanoensembles.

Density functional calculations. Density functional theory calculations were performed using the local density approximation (LDA) [20-22] (discussion of the functional choice is given at the Supplementary Materials Section). The spin-averaged charge density is fitted to plane waves with an energy cut-off of $150 \mathrm{Ha}$, while Kohn-Sham wave functions were constructed using localized Cartesian Gaussian orbital functions ( 50 for Mo, $l \leq 3,28$ for $\mathrm{S}, l \leq 2,38$ for $\mathrm{C}, l \leq 2$, and 12 for $\mathrm{H}, l \leq 1$ ). Relativistic pseudopotentials generated by Hartwigsen, Goedecker, and Hutter [23] were used, with a finite electron Fermi temperature of $0.04 \mathrm{eV}$. Absolute energies were converged to better than $10^{-5} \mathrm{Ha}$. Variable pyrene surface densities were explored using a single pyrene molecule in different hexagonal $\mathrm{MoS}_{2}$ supercells, $2 \times 2\left(\mathrm{Mo}_{4} \mathrm{~S}_{9}\right), 3 \times 3\left(\mathrm{Mog}_{9} \mathrm{~S}_{18}\right), 4 \times 4\left(\mathrm{Mo}_{16} \mathrm{~S}_{32}\right), 6 \times 6\left(\mathrm{Mo}_{36} \mathrm{~S}_{72}\right)$, and $8 \times 8\left(\mathrm{Mo}_{64} \mathrm{~S}_{128}\right)$, 
with results referring to the $8 \times 8$ supercell unless stated otherwise. Gamma-centered k-point meshes generated under the Monkhorst-Pack scheme [24] of $6 \times 6 \times 1,4 \times 4 \times 1,3 \times 3 \times 1,2 \times 2 \times 1$, and $1 \times 1$ $\times 1$ were used for the increasing supercell sizes. The supercell c-axis was fixed at $31.08 \AA$ to avoid interactions between layers. The binding energy of pyrene with $\mathrm{MoS}_{2}$ has been calculated as follows:

$$
E_{\text {bind }}=\left(E_{\text {pyr }}+E_{\mathrm{MoS} 2}\right)-E_{\mathrm{pyr}+\mathrm{MoS} 2}
$$

where $E_{\text {bind }}$ is the binding energy, $E_{\text {pyr+MoS2 }}$ is the energy of the functionalized system, and $E_{\mathrm{pyr}}$ and $E_{\mathrm{MoS} 2}$ are the energies of the isolated pyrene molecule and $\mathrm{MoS}_{2}$ sheet, respectively.

\section{Results and Discussion}

Liquid exfoliated semiconducting $2 \mathrm{H}-\mathrm{MoS}_{2}$ and $2 \mathrm{H}-\mathrm{WS}_{2}$ were prepared following literature procedures. Briefly, bulk $\mathrm{MoS}_{2}$ and $\mathrm{WS}_{2}$ were treated with chlorosulfonic acid to yield exfoliated material of the semiconducting $2 \mathrm{H}$ polytype [1]. A solution of TMDs in DMF is mixed with an excess of pyrene for $36 \mathrm{~h}$, during which the pyrene physisorbs onto the surface of the TMDs. The $\mathrm{MoS}_{2} /$ pyrene and $\mathrm{WS}_{2}$ /pyrene nanoensembles, referred to hereafter as $1 \mathrm{a}$ and $1 \mathrm{~b}$, respectively, were obtained free of non-immobilized pyrene by filtering the reaction mixture over a PTFE filter (100 nm pore size) and subsequently washing the solid residue obtained onto the filter with dichloromethane. The process was followed by monitoring the electronic absorption spectrum of the filtrate until no signatures due to pyrene were observed.

The solubility of $1 \mathrm{a}$ and $1 \mathrm{~b}$ was calculated with the aid of UV-Vis spectroscopy by initially estimating the molar absorptivity as $3.24 \mathrm{~L} \mathrm{~g}^{-1} \mathrm{~cm}^{-1}$ at $400 \mathrm{~nm}$ for $1 \mathrm{a}, 3.04 \mathrm{~L} \mathrm{~g}^{-1} \mathrm{~cm}^{-1}$ at $465 \mathrm{~nm}$ for $1 \mathrm{~b}$ and then applying the Beer-Lambert law. In general, $1 \mathrm{a}$ and $1 \mathrm{~b}$ ensembles present the best solubility in DMF $(100 \mu \mathrm{g} / \mathrm{mL})$, while they also disperse well in o-DCB $(50 \mu \mathrm{g} / \mathrm{mL})$.

Electronic absorption spectroscopy studies were performed in DMF and absorption bands of pyrene at 315, 330, and $345 \mathrm{~nm}$ were detected in both TMD-based $1 \mathrm{a}$ and $1 \mathrm{~b}$ ensembles. Moreover, typical signals of $2 \mathrm{H}-\mathrm{MoS}_{2}$ at 400, 500, 630, and $690 \mathrm{~nm}$ for 1a (Figure 1a) and 2H-WS 2 at 420, 465, 535 , and $640 \mathrm{~nm}$ for $1 \mathrm{~b}$ (Figure $1 \mathrm{~b}$ ) were identified, providing an average of $6-8$ layers. These TMD bands were broadened and red-shifted as compared to those of exfoliated $2 \mathrm{H}-\mathrm{MoS}_{2}$ and $2 \mathrm{H}-\mathrm{WS} 2$, implying ground state electronic interactions between pyrene and the TMDs. Indeed, reversibility is an important parameter and the coated pyrene can be easily removed from the surface of $\mathrm{MoS}_{2}$ and $\mathrm{WS}_{2}$ by washing the ensembles. UV-Vis absorption spectroscopy of the extensively washed 1a and $1 \mathrm{~b}$ by dichloromethane revealed only the bands due to $\mathrm{MoS}_{2}$ and $\mathrm{WS}_{2}$, respectively, without any signature from absorption due to pyrene.

The strong emission of pyrene is a convenient and sensitive probe for identifying itraensemble interactions at the excited states within ensembles $1 \mathrm{a}$ and $1 \mathrm{~b}$. Photoluminescence of monomeric pyrene with fine structure in the $370-430 \mathrm{~nm}$ region was observed for free pyrene upon phtoexcitation at $340 \mathrm{~nm}$ in DMF. Then, after adjusting the optical concentration of $1 \mathrm{a}$ and $1 \mathrm{~b}$ with pyrene at the excitation wavelength, the photoluminescence was measured. It was found that the emission from pyrene was appreciably quenched in $1 \mathrm{a}$ and $1 \mathrm{~b}$ as compared to that of free pyrene (Figure 1c, $\mathrm{d}$, respectively). Evidently, the efficient quenching of fluorescence in 1a and 1a implies strong electronic interactions of non-covalently immobilized pyrene with $\mathrm{MoS}_{2}$ and $\mathrm{WS}_{2}$. In order to further support our results and obtain additional insight on the electronic communication between the individual species within ensembles $1 \mathrm{a}$ and $1 \mathrm{~b}$, time-resolved fluorescence measurements were performed using the time-correlated-single-photon-counting method. The time profile of the fluorescence decay at $396 \mathrm{~nm}$ for the singlet-excited state of free pyrene was monoexponentially fitted with a lifetime of $9.66 \mathrm{~ns}$. Notably, the corresponding fitting for $1 \mathrm{a}$ and $1 \mathrm{~b}$ was biexponential, giving rise to two components, a faster one with lifetime 275 ps, ascribed to efficient interactions of pyrene with $\mathrm{MoS}_{2}$ (238 ps for $\mathrm{WS}_{2}$ ), and a slower one with lifetime $5.36 \mathrm{~ns}$ attributed to non-interacting pyrene. The quenching rate constant $\mathrm{k}_{\mathrm{q}}^{\mathrm{S}}$ for the singlet excited state of pyrene was calculated to be $3.53 \times 10^{9}$ for $\mathrm{MoS}_{2} /$ pyrene $1 \mathrm{a}$ 
and $4.14 \times 10^{9} \mathrm{~s}^{-1}$ for $\mathrm{WS}_{2} /$ pyrene $1 \mathrm{~b}$. The corresponding quantum yield $\Phi_{\mathrm{q}}^{\mathrm{S}}$ was found to be 0.96 and 0.97 , respectively.
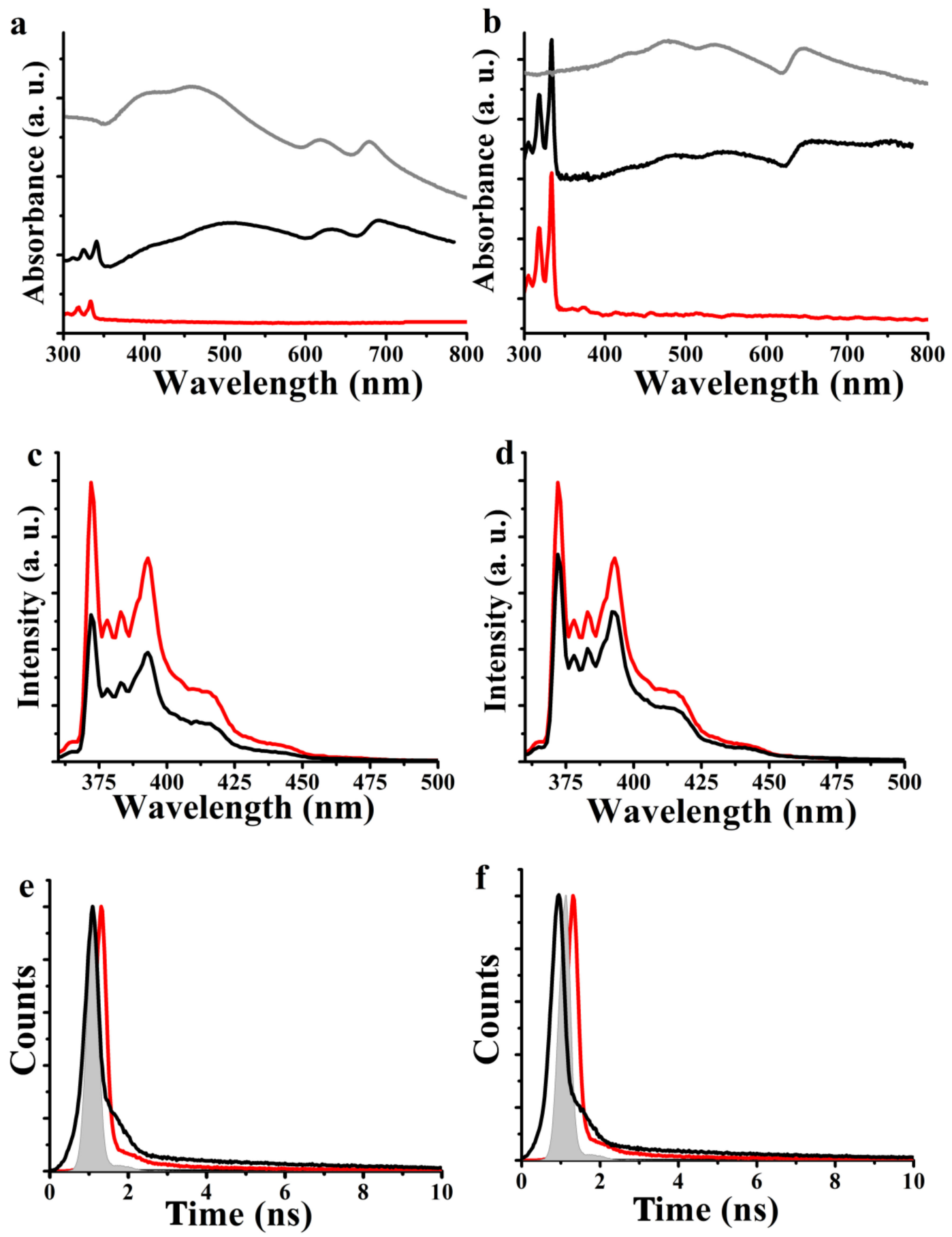

Figure 1. (a,b) UV-Vis spectra for free pyrene (red) as compared with (a) nanoensemble 1a (black line) and exfoliated $\mathrm{MoS}_{2}$ (gray line), and (b) nanoensemble $1 \mathrm{~b}$ (black line) and exfoliated $\mathrm{WS}_{2}$ (gray line), obtained in DMF. (c,d) Photoluminescence spectra for free pyrene (red line) as compared with nanoensemble (c) 1a (black line), and (d) $1 \mathrm{~b}$ (black line), obtained in DMF upon excitation at $340 \mathrm{~nm}$. (e,f) Photoluminescence decay time profiles for free pyrene (red) and nanoensemble (e) 1a (black line), and (f) $1 b$ (black line).

We next explore the pyrene/MoS 2 interaction via density functional theory (DFT) calculations. For comparison we also simulated the two most stable reconstructed 1T-MoS 2 phases found in 
Li-exfoliated samples, i.e., with reconstructed Mo-Mo zig-zag surface chains $\left(1 \mathrm{~T}^{\prime}\right)$ and surface trimmers $\left(1 \mathrm{~T}^{\prime \prime}\right)$ [25]. In all cases, the pyrene binding enthalpies are very close $( \pm 2.5 \mathrm{kcal} / \mathrm{mol})$ and independent of the underlying $\mathrm{MoS}_{2}$ phase, and we report only the $2 \mathrm{H}-\mathrm{MoS}_{2}$ results here. Figure 2 a shows the optimized geometries for pyrene stacked parallel or laterally to the $2 \mathrm{H}-\mathrm{MoS}_{2}$ surface. The parallel-stacked configuration shows a strong binding of $29.7 \mathrm{kcal} / \mathrm{mol}$ whereas the lateral configuration has only $8.3 \mathrm{kcal} / \mathrm{mol}$ binding energy. Thus, we conclude that parallel stacking is the most energetically favorable configuration for pyrene on $2 \mathrm{H}-\mathrm{MoS}_{2}$. We note that the calculated binding energies given here to pristine $2 \mathrm{H}-\mathrm{MoS}_{2}$ are already quite high. Nonetheless, in the presence of surface defects, we would expect this to increase significantly.

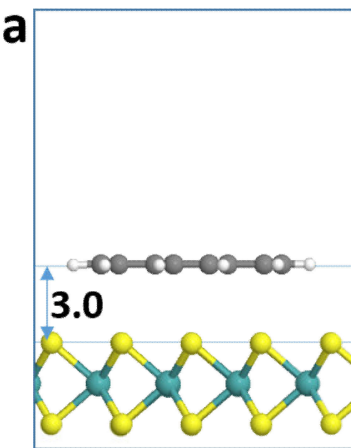

Flat $+29.3$

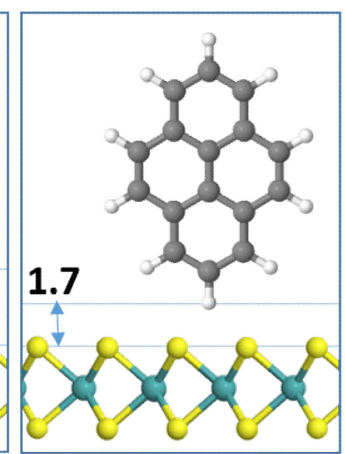

Long-axis vertical

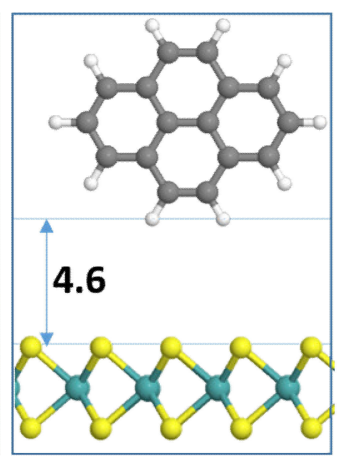

Short-axis vertical
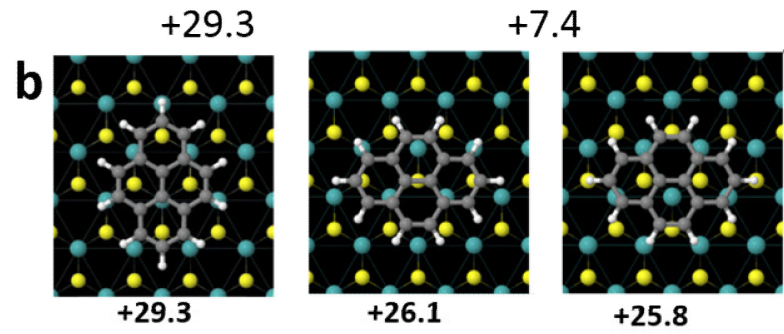

$+25.8$ $+0.4$

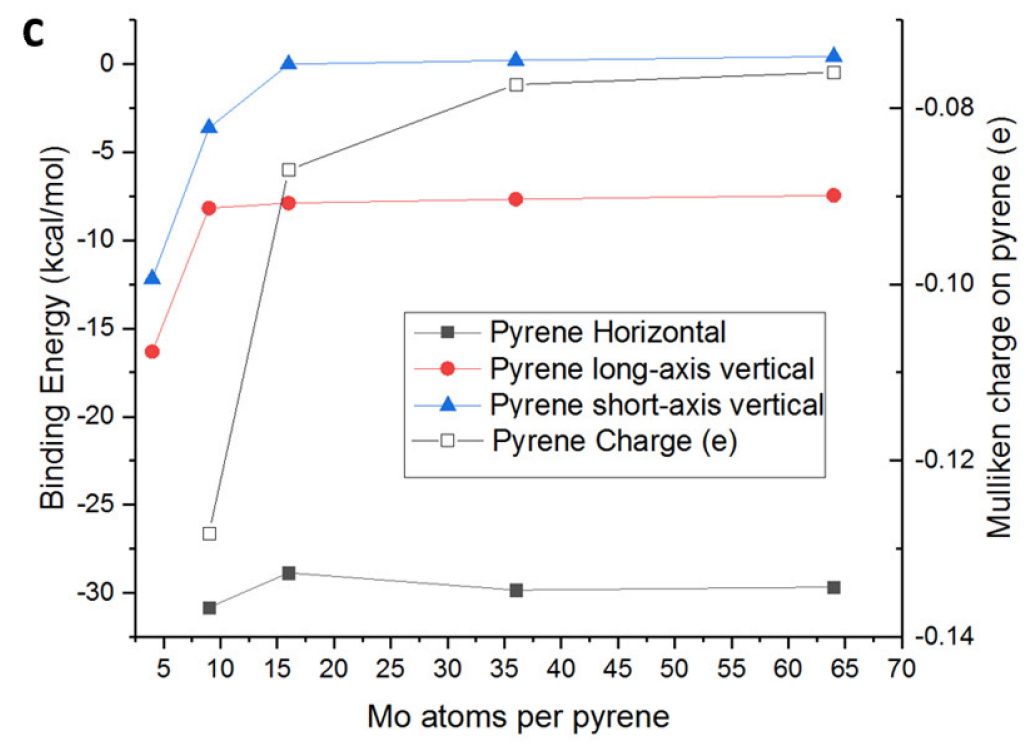

Figure 2. Density functional calculations of pyrene binding to $2 \mathrm{H}-\mathrm{MoS}_{2}$ showing (a) possible stacking orientations of pyrene (binding energies given in $\mathrm{kcal} / \mathrm{mol}$, pyrene/MoS $\mathrm{S}_{2}$ distances in $\AA$ ), (b) variation in binding energy $(\mathrm{kcal} / \mathrm{mol})$ for pyrene in parallel-stacked configuration with surface translation. Atom species are Mo (cyan), S (yellow), C (gray), and H (white), and (c) variation in binding energy per molecule to $\mathrm{MoS}_{2}$ surface for pyrene in different orientations, as a function of surface packing density (filled shapes), right-axis shows Mulliken charge of pyrene in the horizontal configuration (empty squares). 
Closer examination of the precise surface positioning of pyrene is shown in Figure $2 b$, where it can be seen that the most stable position is when the pyrene matches the underlying lattice arrangement, with a sulfur atom positioned beneath each carbon ring. Nonetheless the energy difference remains quite small and at room temperature pyrene is likely to be surface mobile facilitating close-packing arrangements.

To determine the most favorable surface packing density we optimized the three stacking configurations (parallel, long-axis vertical and short-axis vertical) in different size $\mathrm{MoS}_{2}$ supercells $n \times$ $n, n=2,3,4,6$, and 8 . The vertical pyrene configurations are always significantly less stable than the parallel packing, even at high surface packing densities where the molecules can $\pi$-stack with each other. In the most stable parallel configuration the binding energy increases very slightly at $n=3$ (by $2 \mathrm{kcal} / \mathrm{mol}$ ), the maximum possible surface coverage before pyrene overlap. Thus, surface pyrene is most stable at $100 \%$ monolayer packing density, i.e., one pyrene per nine Mo atoms.

Thermogravimetric analysis (TGA) under nitrogen shows a mass loss of $4.5 \%$ and $3.5 \%$ in the temperature range $280-550{ }^{\circ} \mathrm{C}$ for $1 \mathrm{a}$ and $1 \mathrm{~b}$, respectively (Supplementary Materials, Figure S1). This mass loss, associated with pyrene decomposition, allowed us to calculate the immobilization of one pyrene unit per every $21 \mathrm{Mo}$ atoms for 1a and per every $17 \mathrm{~W}$ atoms for $1 \mathrm{~b}$, respectively, representing a rather high coverage. The TGA and DFT results show there would be $100 \%$ monolayer coverage for four-layer $\mathrm{MoS}_{2}$. From the electronic absorption spectroscopy we estimate an average $\mathrm{MoS}_{2}$ flake thickness of six-eight layers and HRTEM confirms that there is typically $<10$ layers, although the sample is heterogeneous and has some thicker flakes. Thus, we conclude that each $\mathrm{MoS}_{2}$ surface is fully saturated with pyrene, with typically two to three layers. This demonstrates the self-organized environmental boundary that pyrene can create on the surface of $\mathrm{MoS}_{2}$ (Figure 3). We note that Mulliken analysis suggests quite a large charge transfer of $0.13 \mathrm{e}$ from $\mathrm{MoS}_{2}$ to pyrene in the ground state (Figure 2c), consistent with the experimental electronic absorption spectroscopy results suggesting ground state electronic interaction between the pyrene and TMDs.

Raman spectroscopy is typically considered a non-invasive characterization technique. However, by varying the incident laser power it is possible to irradiate and possibly modify the sample, while simultaneously obtaining Raman spectra. In this way, we investigated the effect of environmental degradation through local heating and irradiation. Exciting with a $514 \mathrm{~nm}$ laser at less than $0.1 \mathrm{~mW} / 4 \mu \mathrm{m}^{2}$ radiance at ambient conditions, $\mathrm{MoS}_{2} /$ pyrene 1a and exfoliated $\mathrm{MoS}_{2}$ materials all give spectra with the same characteristics. Bands at 378,405 , and $446 \mathrm{~cm}^{-1}$ are associated with the $\mathrm{E}^{1}{ }_{2 \mathrm{~g}}, \mathrm{~A}_{1 \mathrm{~g}}$ and $2 \mathrm{LA}(\mathrm{M})$ modes of $2 \mathrm{H}-\mathrm{MoS}_{2}$, respectively. The absence of characteristic peaks from the metallic polytype $1 \mathrm{~T}-\mathrm{MoS}_{2}\left(\mathrm{~J}_{1}, \mathrm{~J}_{2}\right.$, and $\left.\mathrm{J}_{3}\right)$ at 150,225 , and $325 \mathrm{~cm}^{-1}$ demonstrates the semiconducting nature of $\mathrm{MoS}_{2}$ in the 1a ensemble. Edges and basal plane have different intensity in the $\mathrm{A}_{1 \mathrm{~g}}$ and $\mathrm{E}^{1}{ }_{2 \mathrm{~g}}$ modes of the Raman spectrum of the same flake, yet it has been possible to differentiate the effect on the Raman mode between edges, basal plane and ripplocations in single and well-isolated $\mathrm{MoS}_{2}$ flakes [26]. Although a detailed Raman spectroscopy analysis goes beyond the main aim of our work, a 6.0 and $2.0 \mathrm{~cm}^{-1}$ shift in the $\mathrm{E}_{2 \mathrm{~g}}^{1}$ and $\mathrm{A}_{1 \mathrm{~g}}$ modes for pyrene/MoS $\mathrm{S}_{2}$ versus exfoliated $\mathrm{MoS}_{2}$ are registered. Additionally, $\mathrm{A}_{1 \mathrm{~g}}$ shows more than $100 \%$ intensity enhancement. However, the $\mathrm{E}^{1}{ }_{2 \mathrm{~g}} / \mathrm{A}_{1 \mathrm{~g}}$ intensity ratio is also dependent on other factors e.g., number of layers, thus in our pyrene coated $\mathrm{MoS}_{2}$ and $\mathrm{WS}_{2}$ materials, in which we have an average of few flakes with very different sizes, such information is problematic. Instead, we focus in the $2 \mathrm{LA}(\mathrm{M})$ mode of $\mathrm{MoS}_{2}$ commonly related with sulfur vacancies and defects [27-29]. Upon $633 \mathrm{~nm}$ excitation and due to coupling with the A1 excitonic transition, which produces resonance Raman enhancement of the first and second order vibrational modes, the 2LA(M) mode shows no appreciable changes (Figure S2a), hence proving the non-covalent interactions of pyrene with $\mathrm{MoS}_{2}$. Overall, such changes in the Raman modes could be due to the development of electronic interactions between pyrene and $\mathrm{MoS}_{2}$ in the ensemble material and/or reduction of the number of $\mathrm{MoS}_{2}$ layers resulted by the immobilization of pyrene. Similar is the situation with $\mathrm{WS}_{2}$ as we observe a $3.3 \mathrm{~cm}^{-1}$ shift in the $\mathrm{A}_{1 \mathrm{~g}}$ mode for pyrene/ $\mathrm{WS}_{2}$ versus the value registered for exfoliated $\mathrm{WS}_{2}$. Moreover, changes in the bandwidth have been also observed, 
e.g., $6 \mathrm{~cm}^{-1}$ reduction of the $\mathrm{E}^{1}{ }_{2 \mathrm{~g}}+2 \mathrm{LA}(\mathrm{M})$ modes of pyrene/WS $\mathrm{W}_{2}$ as compared to exfoliated $\mathrm{WS}_{2}$ (Figure S2b).

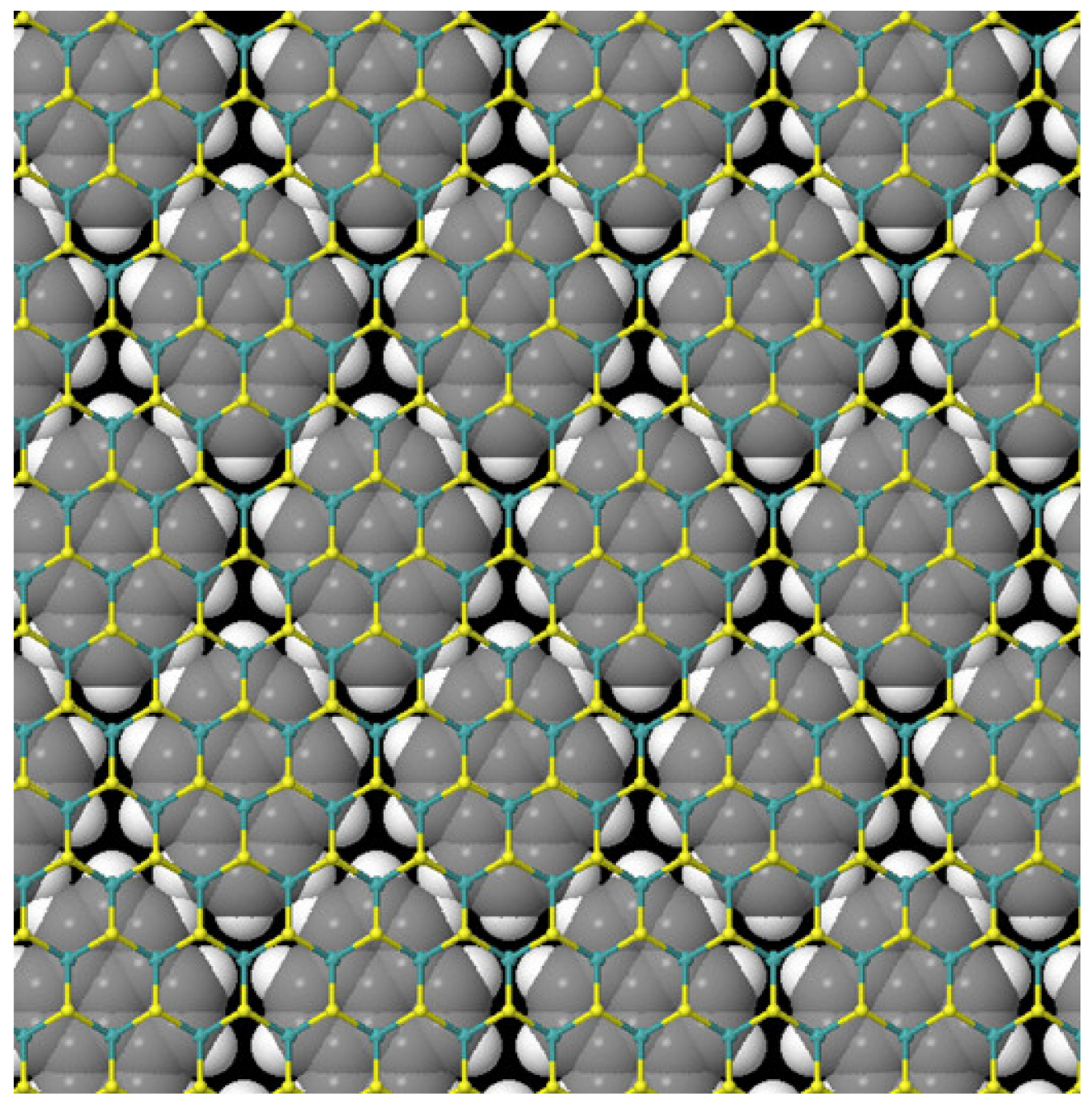

Figure 3. Calculated structure for pyrene in a $\mathrm{MoS}_{2}-3 \times 3$ surface array at $100 \%$ surface coverage, the most stable packing configuration. Pyrene atoms are shown with atomic van der Waals radii, showing the complete surface protective layer. Experimentally we calculate pyrene is forming between two and three layers.

However, the situation dramatically changes once the laser radiance is increased to $0.9 \mathrm{~mW} / 4 \mu \mathrm{m}^{2}$. In addition to a frequency downshift of the previous $\mathrm{E}_{2 \mathrm{~g}}^{1}, \mathrm{~A}_{1 \mathrm{~g}}$ and $2 \mathrm{LA}(\mathrm{M})$ modes, due to laser heating of the sample, new and strong Raman bands appear at a range of energies coming from oxidized states of Mo (Figure 4a). Well-resolved bands appear in the spectrum of exfoliated $\mathrm{MoS}_{2}$ at 843, 895, and $948 \mathrm{~cm}^{-1}$ due to oxidized Mo [30,31]. In addition, the frequency of broad features at 151, 285, and $340 \mathrm{~cm}^{-1}$ are reminiscent of $\mathrm{MoO}_{3}$ phases [30,31], however, the deviation from crystalline $\mathrm{MoO}_{3}$ in relative peak intensities at higher frequencies $\left(600-1000 \mathrm{~cm}^{-1}\right)$ suggests a more disordered structure $\left(\mathrm{MoO}_{\mathrm{x}}\right)$. The $948 \mathrm{~cm}^{-1}$ peak is likely attributable to either $\mathrm{Mo}=\mathrm{O}$ or Mo-peroxide stretching modes. These peaks demonstrate the rapid photodegradation of the material upon light irradiation under ambient conditions. Yet remarkably, when $\mathrm{MoS}_{2} /$ pyrene 1a is subjected to the same exposure conditions (514 $\mathrm{nm}$ with $0.9 \mathrm{~mW} / 4 \mu \mathrm{m}^{2}$ ) no perturbation of the $\mathrm{MoS}_{2}$ is observed, the Raman spectrum retaining its original features (Figure $4 b$ ). 

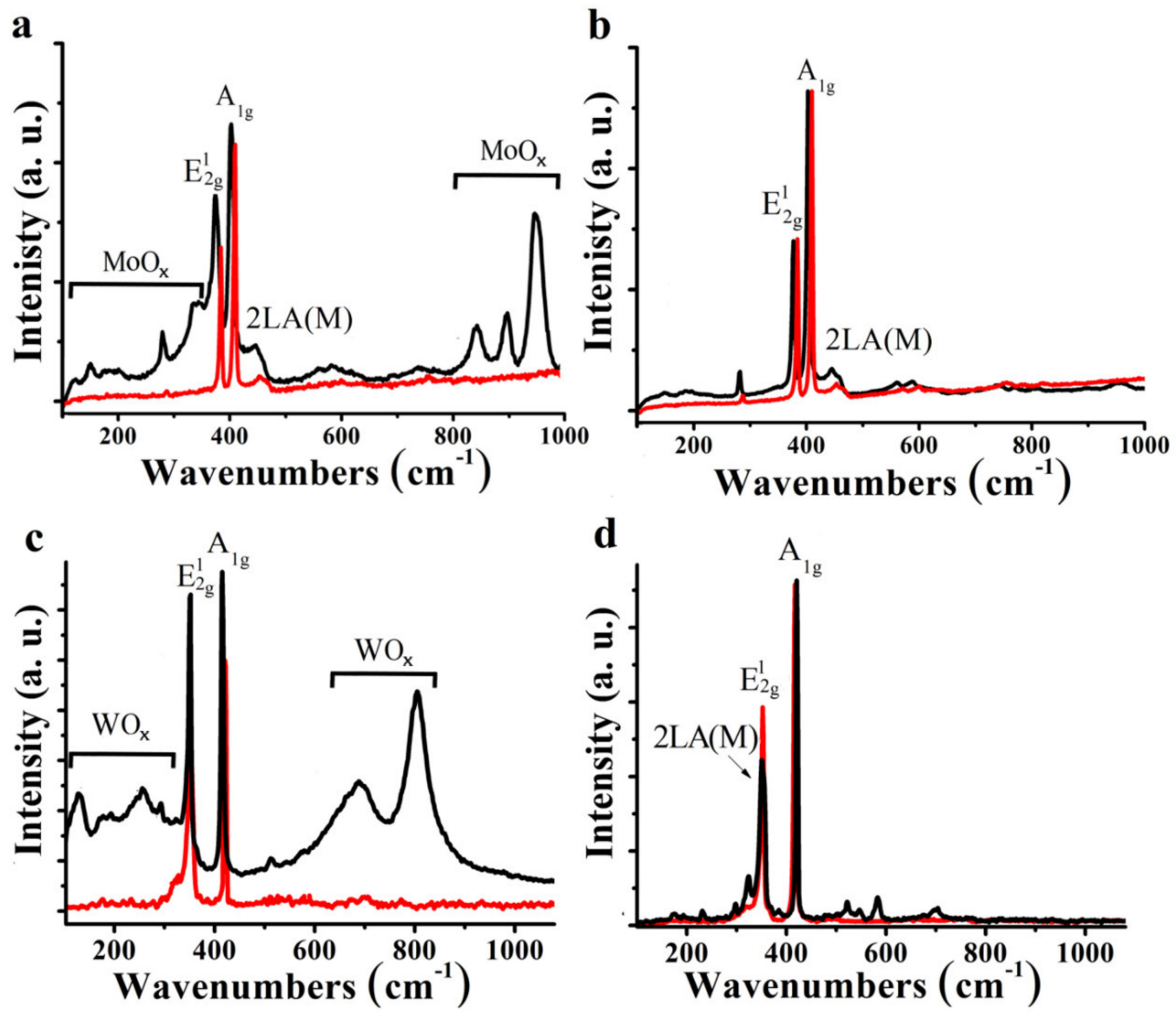

Figure 4. Raman spectra acquired at ambient conditions upon excitation at $514 \mathrm{~nm}$ with $0.1 \mathrm{~mW} / 4 \mu \mathrm{m}^{2}$ (red line) and $0.9 \mathrm{~mW} / 4 \mu^{2}$ (black line) laser radiance for (a) exfoliated $\mathrm{MoS}_{2}$, (b) $\mathrm{MoS}_{2} /$ pyrene 1a, (c) exfoliated $\mathrm{WS}_{2}$, and (d) $\mathrm{WS}_{2} /$ pyrene $1 \mathrm{~b}$. The $\mathrm{E}_{2 \mathrm{~g}}^{1}, \mathrm{~A}_{1 \mathrm{~g}}$, and $2 \mathrm{LA}(\mathrm{M})$ peaks in the black spectra are downshifted due to laser heating.

A similar situation occurs for $\mathrm{WS}_{2} /$ pyrene $1 \mathrm{~b}$ and exfoliated $\mathrm{WS}_{2}$. Under resonance conditions with green laser illumination ( $514 \mathrm{~nm}$, with less than $0.1 \mathrm{~mW} / 4 \mu \mathrm{m}^{2}$ radiance at ambient conditions) three intense and distinguishable bands at 350,355 , and $418 \mathrm{~cm}^{-1}$, due to the $2 \mathrm{LA}(\mathrm{M}), \mathrm{E}^{1}{ }_{2 \mathrm{~g}}$ and $\mathrm{A}_{1 \mathrm{~g}}$ modes, respectively, are visible (Figure 4c). However, upon increasing the laser radiance to $0.9 \mathrm{~mW} / 4 \mu \mathrm{m}^{2}$, exfoliated $\mathrm{WS}_{2}$ showed additional Raman peaks at 127, 257, and $294 \mathrm{~cm}^{-1}$ related to somewhat disordered $\mathrm{WO}_{\mathrm{x}}$ as well as two sharp peaks at 688 and $805 \mathrm{~cm}^{-1}$ (Figure 4c) resembling partially hydrated $\mathrm{h}-\mathrm{WO}_{3}$ [32]. All those bands associated with $\mathrm{WO}_{\mathrm{x}}$ species were absent when $\mathrm{WS}_{2} /$ pyrene $1 \mathrm{~b}$ was exposed to light irradiation, even at higher laser radiance (Figure $4 \mathrm{~d}$ ). It should also be noted the proportional decrease in the $\mathrm{MoS}_{2}$ and $\mathrm{WS}_{2}$ characteristic signals $\mathrm{E}_{2 \mathrm{~g}}^{1}, \mathrm{~A}_{1 \mathrm{~g}}$, and $2 \mathrm{LA}(\mathrm{M})$, according to the degree of oxidation. Consequently, pyrene plays an important protecting role of $\mathrm{MoS}_{2}$ and $\mathrm{WS}_{2}$ from oxidation.

In order to investigate in more detail at the local scale the pyrene immobilization onto the TMD materials, a different TEM analysis was performed. These kinds of analyses are required to have access to this structural and chemical information at the (sub-)nanometer level [27,33-36]. Figure 5a,c shows STEM high-angular dark-field (HAADF) images of two flakes of $\mathrm{MoS}_{2} /$ pyrene 1a (Figure 5a) and exfoliated $\mathrm{MoS}_{2}$ (Figure 5c), both after laser-irradiation $\left(0.9 \mathrm{~mW} / 4 \mu \mathrm{m}^{2}\right)$ at ambient conditions. Two EEL line spectra (LS) were acquired following the green lines marked on the micrographs. For each sample two different regions, marked as (i) and (ii) in each of the images were selected. The spectra in Figure $5 \mathrm{~b}$ for $1 \mathrm{a}$ show $\mathrm{S}-\mathrm{L}_{2,3}, \mathrm{Mo}-\mathrm{M}_{4,5}, \mathrm{C}-\mathrm{K}$, and Mo- $\mathrm{M}_{2,3}$ edges. The Mo and $\mathrm{S}$ edges correspond to the $\mathrm{MoS}_{2}$ flake and the carbon content confirms the presence of the pyrene moieties [28,35,36]. Remarkably, 
examining close to fifteen different areas of different flakes, no oxygen was detected in $\mathrm{MoS}_{2} /$ pyrene ensemble 1a. However, this was not the case for exfoliated $\mathrm{MoS}_{2}$ without any pyrene immobilized on it, where oxygen was easily detected (Figure $5 c, d$ ). This oxygen (see Figure $5 d$-iii) corresponds to the oxidation of the $\mathrm{MoS}_{2}$ flake, where a mix of molybdenum oxide and molybdenum disulfide can be observed. This conclusion can be clearly inferred from a comparison of the S- $\mathrm{L}_{2,3}$ edge in the two different selected regions of the LS of Figure $5 c$. The $S-L_{2,3}$ edge of the EEL spectrum in Figure $5 d$-iii is similar to that obtained by others investigating mixed $\mathrm{MoO}_{\mathbf{x}}-\mathrm{MoS}_{2}$ phases in nanomaterials [37]. Similar imaging assays have been performed for $\mathrm{WS}_{2} /$ pyrene $1 \mathrm{~b}$ (Figure S3).
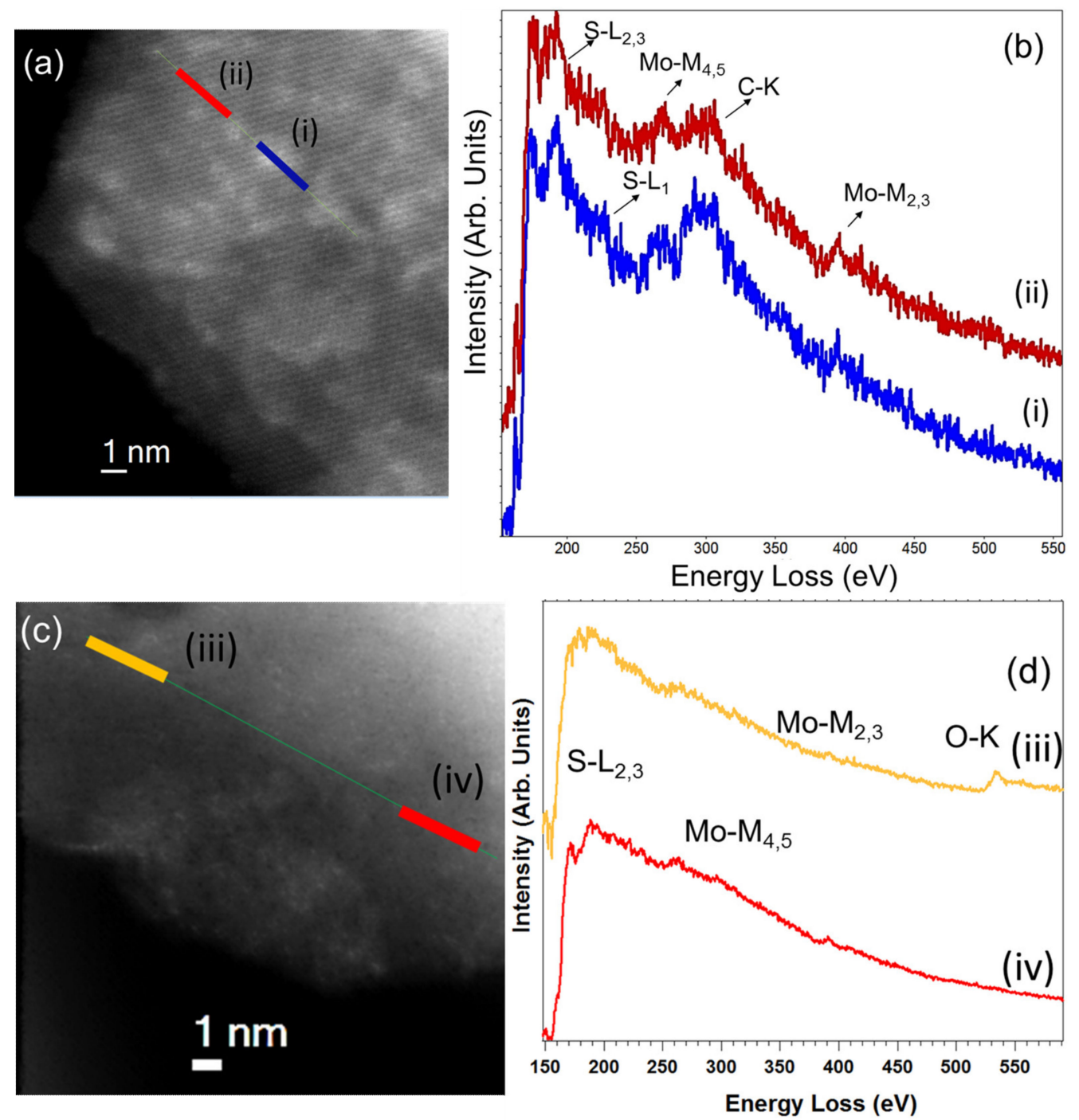

Figure 5. (a,c) High-angular dark-field (HAADF)-STEM micrographs of $\mathrm{MoS}_{2} /$ pyrene 1a and exfoliated $\mathrm{MoS}_{2}$ flakes, respectively. Two electron energy loss spectroscopy (EELS) line spectra were recorded on these flakes, see the green lines marked in Figure 5a,c. (b) Two EEL spectra corresponding to the sum of seven spectra recorded in each of the two areas highlighted in red (i) and blue (ii) in Figure 5a, respectively. Carbon, corresponding to pyrene, sulfur, and molybdenum (associated to $\mathrm{MoS}_{2}$ ) are detected in these spectra. (d) Two EEL spectra corresponding to the addition of eight spectra collected in each of the two regions highlighted in orange (iii) and red (iv) in Figure 5c, respectively. The presence of oxygen denotes the clear oxidation of this $\mathrm{MoS}_{2}$ flake, see text for details.

Shedding light on the oxidation mechanism, initially, oxygen from the air is physisorbed onto the exfoliated $\mathrm{MoS}_{2}$ and $\mathrm{WS}_{2}$. Upon laser irradiation, the surface of the TMDs is damaged, with defective 
sites created and stabilized by chemisorbed oxygen. Sulfur atoms may leave the lattice and be substituted by oxygen [38-40], resulting in structural deformation, as well as hole formation whose edges can become oxygen saturated. Localized heating, due to Raman laser irradiation conditions, further damages the chemisorbed TMDs surface, generating abundant structural defects and etching the surface. The result of such processes are visible in the Raman spectra of exfoliated $\mathrm{MoS}_{2}$ and $\mathrm{WS}_{2}$ after prolonged irradiation period, where not only new bands due to $\mathrm{MoO}_{\mathrm{x}}$ and $\mathrm{WO}_{\mathrm{x}}$ appear, while the intensity of the characteristic $\mathrm{A}_{1 \mathrm{~g}}, \mathrm{E}^{1}{ }_{2 \mathrm{~g}}$, and 2LA(M) modes decreases.

At the same time, interaction with surface water and subsequent degradation is an important process that can also be blocked by surface pyrene. The surface of TMDs is intrinsically mildly hydrophilic, due to both the transition metal and the chalcogen atoms. Moreover, doping TMDs with oxygen results in a more hydrophilic surface [41], susceptible to hydrogen-bonding interactions with moisture from air. Then, the presence of Mo-edge-defected $\mathrm{MoS}_{2}$ structures (or W for $\mathrm{WS}_{2}$ ) results in moisture air-induced transformations of sulfur to sulfate species, eventually leading to $\mathrm{MoO}_{\mathrm{x}}$ and $\mathrm{WO}_{\mathrm{x}}$ accompanied by the liberation of sulfuric acid via a series of oxidation and replacement reactions [42]. Conversely, adsorption of hydrophobic hydrocarbons onto TMDs renders the surface layer hydrophobic [43]. Hence, physisorption of pyrene onto $\mathrm{MoS}_{2}$ and $\mathrm{WS}_{2}$ acts as barrier, insulating TMDs from the outer environment and prevents the initiation of surface reactions towards vacancies creation, sulfate/sulfuric acid species generation, substitution of sulfur by oxygen, etc., which are catalyzed by humid air and assisted by laser irradiation. Furthermore, pyrene is likely to bind even more strongly to defective sites than pristine basal material, further driving its barrier capability.

Exfoliated $\mathrm{MoS}_{2}$ and $\mathrm{WS}_{2}$ stored in the dark for more than two years in powder form and in the presence of air show a non-depreciated enhancement in the oxidation Raman bands. In fact, $\mathrm{WS}_{2}$ was found to be more susceptible to oxidation as compared to $\mathrm{MoS}_{2}$, which can be rationalized by considering the higher number of electrons present in $\mathrm{W}$ as compared to those in Mo. Interestingly, those oxidation features are discernible, but to a lower extent (Figure 6), even in freshly exfoliated TMDs and according to a couple of very recent articles this is due to defective passivation with oxygen under air exposure in ambient conditions [44,45]. Then, coated pyrene plays a protective role, as after two years no obvious change in the Raman spectra for $1 \mathrm{a}$ and $1 \mathrm{~b}$ is observed (Figure 6). In sharp contrast, freshly exfoliated TMDs, without coated pyrene, show an appreciable oxidation effect after two years in the dark and in the presence of air. First, enhancement of the oxidation Raman bands for around $70 \%$ and more than $100 \%$ in $\mathrm{MoS}_{2}$ and $\mathrm{WS}_{2}$ respectively, is identified. Then, more relevant is the presence of new features at 921 and $1002 \mathrm{~cm}^{-1}$ due to oxidation in the aged exfoliated $\mathrm{MoS}_{2}$ and 233 and $765 \mathrm{~cm}^{-1}$ in the aged exfoliated $\mathrm{WS}_{2}$. In addition, the shift for some of the oxidation Raman modes, such as 1 and $4 \mathrm{~cm}^{-1}$ at 282 and $813 \mathrm{~cm}^{-1}$ in fresh $\mathrm{MoS}_{2}$ and 3, 8, and $10 \mathrm{~cm}^{-1}$ at 514, 754, and $764 \mathrm{~cm}^{-1}$ in fresh $\mathrm{WS}_{2}$, clearly show evolution in the oxidative species. Overall, we note that materials kept for more than two years remain structurally unaltered, as demonstrated in the Raman spectra of $1 \mathrm{a}$ and $1 \mathrm{~b}$ (Figure 6), showing that pyrene not only blocks oxidation but also prevents aging of TMDs $[12,16]$. 

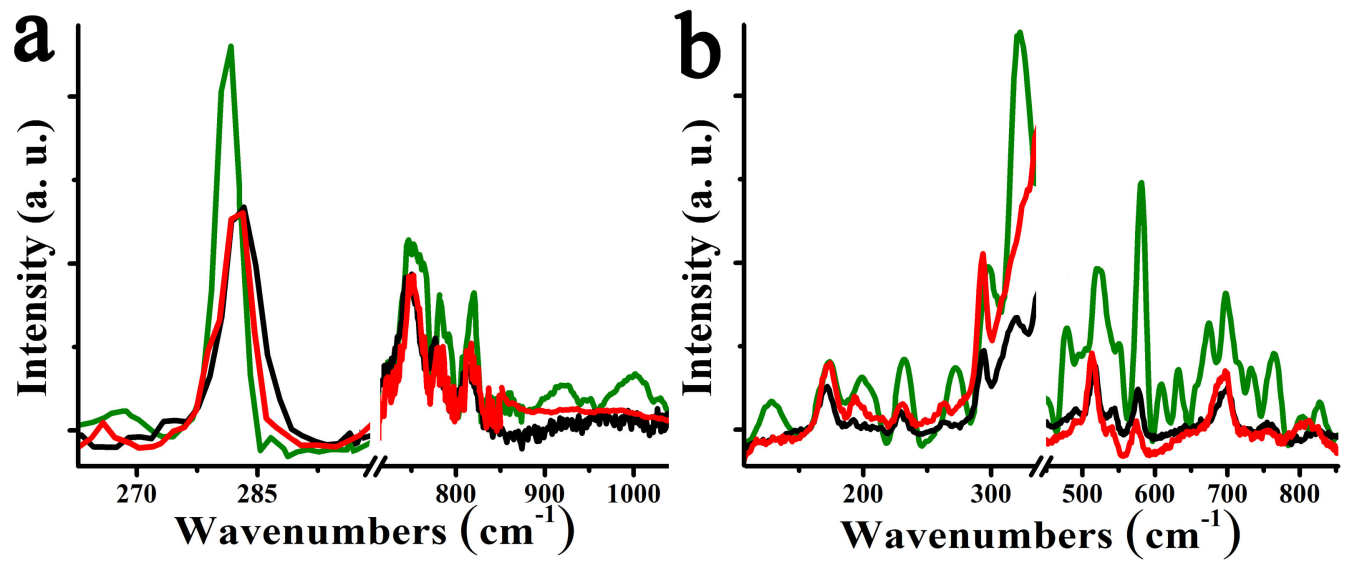

Figure 6. Raman spectra at $514 \mathrm{~nm}$ with $0.1 \mathrm{~mW} / 4 \mu \mathrm{m}^{2}$ laser radiance, for (a) fresh exfoliated $\mathrm{MoS}_{2}$ (black), aged exfoliated $\mathrm{MoS}_{2}$ (green) and aged $\mathrm{MoS}_{2} /$ pyrene 1a (red), and (b) fresh exfoliated $\mathrm{WS}_{2}$ (black), aged exfoliated $\mathrm{WS}_{2}$ (green) and aged $\mathrm{WS}_{2} /$ pyrene $1 \mathrm{~b}$ (red). The region showing the characteristic Raman bands due to $\mathrm{MoS}_{2}$ and $\mathrm{WS}_{2}$ have deliberately been omitted to allow better observation of the bands due to $\mathrm{MoO}_{\mathrm{x}}$ and $\mathrm{WO}_{\mathrm{x}}$.

\section{Conclusions}

Pyrene has been demonstrated to bind to the basal plane of $\mathrm{MoS}_{2}$ and $\mathrm{WS}_{2}$ via multiple $\pi-\mathrm{S}$ interactions, yielding a close-packed continuous surface coverage with significant ground-state charge-transfer. This simple non-destructive and reversible process has the advantage of non-covalent modification, leaving intact and undisturbed the surface of TMDs, without permanently affecting their electronic and mechanical properties, in contrast with covalent bonding incorporation of other photoactive species [29,46-50].

The resultant dense pyrene coating has been shown using Raman spectroscopy to shield the surface of TMDs from environmental degradation under ambient conditions (air, moisture, and light illumination), without requiring isolation of the material from the environment, minimizing necessity for high-cost equipment and advanced processes for handling the materials. The additional beneficial role of pyrene for inhibiting TMD aging was demonstrated via unaltered Raman spectroscopy of protected samples stored under ambient conditions for two years. This simple and effective oxidation resistance route should also be applicable to other TMDs and 2D-layered materials, such as $\mathrm{HfSe}_{2}$ which is known to be particularly susceptible to oxidation [51].

Supplementary Materials: The following are available online at http://www.mdpi.com/2079-4991/10/2/363/s1, Figure S1: TGA of (a) 1a (red line) as compared with exfoliated $\mathrm{MoS}_{2}$ of the semiconducting polytype (black line), (b) and $1 \mathrm{~b}$ (red line) as compared with exfoliated $\mathrm{WS}_{2}$ of the semiconducting polytype (black line) under nitrogen atmosphere.; Figure S2: Raman spectra (a) at $633 \mathrm{~nm}$ with $0.1 \mathrm{~mW} / 4 \mu \mathrm{m}^{2}$ laser radiance, for exfoliated $\mathrm{MoS}_{2}$ (black) and $\mathrm{MoS}_{2} /$ pyrene $1 \mathrm{~b}(\mathrm{red})$, and (b) at $514 \mathrm{~nm}$ with $0.1 \mathrm{~mW} / 4 \mu \mathrm{m}^{2}$ laser radiance, for exfoliated $\mathrm{WS}_{2}$ (black) and $\mathrm{WS}_{2} /$ pyrene $1 \mathrm{~b}$ (red); Figure S3: (a,c) HAADF-STEM micrographs of $\mathrm{WS}_{2} /$ pyrene $1 \mathrm{~b}$ and exfoliated $\mathrm{WS}_{2}$ flakes, respectively. An EELS spectrum-image and an EELS spectrum-line have been recorded on these respective flakes, see the green line marked in Figure S3c. (b) Two EEL spectra corresponding to the sum of fourteen spectra recorded in each of the two areas highlighted in red (i) and blue (ii) in Figure S3a, respectively. Carbon, corresponding to pyrene and sulfur (associated to $\mathrm{WS}_{2}$ ) are detected in these spectra. (d) Two EEL spectra corresponding to the addition of twelve spectra collected in each of the two regions highlighted in orange (iii) and red (iv) in Figure S3c, respectively. The presence of oxygen denotes the clear oxidation of this $\mathrm{WS}_{2}$ flake, as it was the case for $\mathrm{MoS}_{2}$ (see Figure 5 in the paper); Table S1: Comparison of calculated structural parameters and cohesion energies for $2 \mathrm{H}-\mathrm{MoS}_{2}$ with different approximations and the corresponding experimental data (Exp.). The LDA optimisations have been performed allowing atoms and lattice parameter to change in a hexagonal lattice using a $24 \times 24 \times 12 \mathrm{MP} k$-point grid. The cohesion energy $\left(\Delta \mathrm{E}_{\mathrm{coh}}\right)$ is taken as the energy difference between isolated $S$ and Mo atoms and $\mathrm{MoS}_{2}$ normalized per atom. For the isolated atoms spin-polarised calculations with a single $k$-point at $\Gamma$ have been performed, while $\mathrm{MoS}_{2}$ has been calculated with a spin-averaged approach; Table S2: Binding energies $(\mathrm{eV})$ for the adsorption of benzene and naphthalene on the surface of $\mathrm{MoS}_{2}$ calculated using different methods. Our calculations have been performed on an $8 \times 8$ supercell of $\mathrm{MoS}_{2}$. The binding energy is 
taken as the difference in energy between the combined system $\left(\mathrm{MoS}_{2}+\right.$ molecule) and the energy of the separate components. Both $\mathrm{MoS}_{2}$ and the isolated molecules have been calculated using a single $k$-point at $\Gamma$.

Author Contributions: Conceptualization, N.T.; Methodology, N.T. and C.P.E.; Calculations, C.P.E. and Y.S.-A.-B; Investigation, R.C.-V. and Y.S.-A.-B; Microscopy and spectro-microscopy analysis, R.A. Spectroscopy B.H., Y.S.-A.-B., R.C.-V. Writing-Original draft preparation, N.T., R.C.-V., Y.A.-S.-B. and C.P.E.; Writing-Review and editing, all authors; Supervision, C.P.E., B.H. and N.T. All authors have read and agreed to the published version of the manuscript.

Funding: This research was funded by the European Union's Horizon 2020 research and innovation programme under the Marie Sklodowska-Curie grant agreement No 642742, under the "Graphene Flagship" project grant agreement No 785219 and under the ESTEEM-3 project grant agreement No 823717. This research was also partially funded by the project "Advanced Materials and Devices" (MIS 5002409), which is implemented under the "Action for the Strategic Development on the Research and Technological Sector" funded by the Operational Program "Competitiveness, Entrepreneurship and Innovation" (NSRF 2014-2020) and co-financed by Greece and the European Union (European Regional Development Fund). This work was supported by the COST Action CA15107 MultiComp. This research was also supported by the Spanish Ministerio de Economia y Competitividad (MAT2016-79776-P), from the Government of Aragon and the European Social Fund under the project “Construyendo Europa desde Aragon" 2014-2020 (grant number E13_17R).

Acknowledgments: The SR-EELS studies were conducted at the Laboratorio de Microscopias Avanzadas, Instituto de Nanociencia de Aragon, Universidad de Zaragoza, Spain. Some of the calculations were performed using the Centre de Calculs Intensif Pays de la Loire (CCIPL).

Conflicts of Interest: The authors declare no conflict of interest.

\section{References}

1. Pagona, G.; Bittencourt, C.; Arenal, R.; Tagmatarchis, N. Exfoliated Semiconducting Pure $2 \mathrm{H}-\mathrm{MoS} 2$ and $2 \mathrm{H}-\mathrm{WS}_{2}$ Assisted by Chlorosulfonic Acid. Chem. Commun. 2015, 51, 12950-12953. [CrossRef]

2. Fan, X.; Xu, P.; Li, Y.C.; Zhou, D.; Sun, Y.; Nguyen, M.A.T.; Terrones, M.; Mallouk, T.E. Controlled Exfoliation of $\mathrm{MoS}_{2}$ Crystals into Trilayer Nanosheets. J. Am. Chem. Soc. 2016, 138, 5143-5149. [CrossRef]

3. Niu, L.; Coleman, J.N.; Zhang, H.; Shin, H.; Chhowalla, M.; Zheng, Z. Production of Two-Dimensional Nanomaterials Via Liquid-Based Direct Exfoliation. Small 2016, 12, 272-293. [CrossRef]

4. Grayfer, E.D.; Kozlova, M.N.; Fedorov, V.E. Colloidal 2D Nanosheets of $\mathrm{MoS}_{2}$ and Other Transition Metal Dichalcogenides Through Liquid-Phase Exfoliation. Adv. Colloid Interface Sci. 2017, 245, 40-61. [CrossRef]

5. Chow, P.K.; Jacobs-Gedrim, R.B.; Gao, J.; Lu, T.-M.; Yu, B.; Terrones, H.; Koratkar, N. Defect-Induced Photoluminescence in Monolayer Semiconducting Transition Metal Dichalcogenides. ACS Nano 2015, 9, 1520-1527. [CrossRef]

6. Tongay, S.; Zhou, J.; Ataca, C.; Liu, J.; Kang, J.S.; Matthews, T.S.; You, L.; Li, J.; Grossman, J.C.; Wu, J. Broad-Range Modulation of Light Emission in Two-Dimensional Semiconductors by Molecular Physisorption Gating. Nano Lett. 2013, 13, 2831-2836. [CrossRef]

7. Pritchard, C.; Midgley, J.W. The Effect of Humidity on the Friction and Life of Unbonded Molybdenum Disulfide Films. Wear 1969, 13, 39-50. [CrossRef]

8. Wang, Z.; Zhang, Y.-J.; Liu, M.; Peterson, A.; Hurt, R.H. Oxidation Suppression During Hydrothermal Phase Reversion Allows Synthesis of Monolayer Semiconducting $\mathrm{MoS}_{2}$ in Stable Aqueous Suspension. Tribol. Lett. 2014, 53, 329-336. [CrossRef]

9. Tagawa, M.; Muromoto, M.; Hachiue, S.; Yokota, K.; Ohmae, N.; Matsumoto, K.; Suzuki, M. Hyperthermal Atomic Oxygen Interaction with $\mathrm{MoS}_{2}$ Lubricants and Relevance to Space Environmental Effects in Low Earth Orbit-Effects on Friction Coefficient and Wear-Life. Tribol. Lett. 2005, 18, 437-443. [CrossRef]

10. Liang, T.; Sawyer, W.G.; Perry, S.S.; Sinnott, S.B.; Phillpot, S.R. Energetics of Oxidation in $\mathrm{MoS}_{2}$ Nanoparticles by Density Functional Theory. J. Phys. Chem. C 2011, 115, 10606-10616.

11. Liang, T.; Sawyer, W.G.; Perry, S.S.; Sinnott, S.B.; Phillpot, S.R. First-Principles Determination of Static Potential Energy Surfaces for Atomic Friction in $\mathrm{MoS}_{2}$ and $\mathrm{MoO}_{3}$. Phys. Rev. B 2008, 77, 104105. [CrossRef]

12. Gao, J.; Li, B.; Tan, J.; Chow, P.; Lu, T.-M.; Koratkar, N. Aging of Transition Metal Dichalcogenide Monolayers. ACS Nano 2016, 10, 2628-2635. [CrossRef] [PubMed]

13. Leonhardt, A.; Chiappe, D.; Asselberghs, I.; Radu, I.; de Gendt, S. Improving MOCVD MoS 2 Electrical Performance: Impact of Minimized Water and Air Exposure Conditions. IEEE Electron. Dev. Lett. 2017, 38, 1606-1609. [CrossRef] 
14. Lee, G.-H.; Cui, X.; Kim, Y.D.; Arefe, G.; Zhang, X.; Lee, C.-H.; Ye, F.; Watanabe, K.; Taniguchi, T.; Kim, P.; et al. Highly Stable, Dual-Gated $\mathrm{MoS}_{2}$ Transistors Encapsulated by Hexagonal Boron Nitride with Gate-Controllable Contact, Resistance, and Threshold Voltage. ACS Nano 2015, 9, 7019-7026. [CrossRef]

15. Ahn, S.; Kim, G.; Nayak, P.K.; Yoon, S.I.; Lim, H.; Shin, H.-J.; Shin, H.S. Prevention of Transition Metal Dichalcogenide Photodegradation by Encapsulation with h-BN Layers. ACS Nano 2016, 10, 8973-8979. [CrossRef]

16. Kim, S.-Y.; Kwak, J.; Kim, J.H.; Lee, J.-U.; Jo, Y.; Kim, S.Y.; Cheong, H.; Lee, Z.; Kwon, S.-Y. Substantial Improvements of Long-Term Stability in Encapsulation-Free $\mathrm{WS}_{2}$ Using Highly Interacting Graphene Substrate. 2D Mater. 2017, 4, 011007. [CrossRef]

17. Wood, J.D.; Wells, S.A.; Jariwala, D.; Chen, K.; Cho, E.; Sangwan, V.K.; Liu, X.; Lauhon, L.J.; Marks, T.J.; Hersam, M.C. Effective Passivation of Exfoliated Black Phosphorus Transistors Against Ambient Degradation. Nano Lett. 2014, 14, 6964-6970. [CrossRef]

18. Jeanguillaume, C.; Colliex, C. Spectrum-Image: The Next Step in EELS Digital Acquisition and Processing. Ultramicroscopy 1989, 28, 252-257. [CrossRef]

19. Arenal, A.; de la Peña, F.; Stephan, O.; Walls, M.; Tence, M.; Loiseau, A.; Colliex, C. Extending the Analysis of EELS Spectrum-Imaging Data, From Elemental to Bond Mapping in Complex Nanostructures. Ultramicroscopy 2008, 109, 32-38. [CrossRef]

20. Briddon, P.; Jones, R. LDA Calculations Using a Basis of Gaussian Orbitals. Phys. Status Sol. B 2000, 217, 131-171. [CrossRef]

21. Rayson, M.; Briddon, P. Highly Efficient Method for Kohn-Sham Density Functional Calculations of 500-10,000 Atom Systems. Phys. Rev. B 2009, 80, 205104-205115. [CrossRef]

22. Briddon, P.R.; Rayson, M.J. Accurate Kohn-Sham DFT with the Speed of Tight Binding: Current Techniques and Future Directions in Materials Modelling. Phys. Status Sol. B 2011, 248, 1309-1318. [CrossRef]

23. Hartwigsen, C.; Gøedecker, S.; Hutter, J. Relativistic Separable Dual-Space Gaussian Pseudopotentials from H to Rn. Phys. Rev. B 1998, 58, 3641-3662. [CrossRef]

24. Monkhorst, H.J.; Pack, J.D. Special Points for Brillouin-zone Integrations. Phys. Rev. B 1976, 13, 5188-5192. [CrossRef]

25. Chou, S.S.; Sai, N.; Lu, P.; Coker, E.N.; Liu, S.; Artyushkova, K.; Luk, T.S.; Kaehr, B.; Brinker, C.J. Understanding Catalysis in a Multiphasic Tw-Dimensional Transition Metal Dichalcogenide. Nat. Commun. 2015, 6, 8311. [CrossRef] [PubMed]

26. Fabbri, F.; Rotunno, E.; Cinquanta, E.; Campi, D.; Bonnini, E.; Kaplan, D.; Lazzarini, L.; Bernasconi, M.; Ferrari, C.; Longo, M.; et al. Novel Near-Infrared Emission from Crystal Defects in $\mathrm{MoS}_{2}$ Multilayer Flakes. Nat. Commun. 2016, 7, 13044. [CrossRef]

27. Vallan, L.; Canton-Vitoria, R.; Gobeze, H.B.; Jang, Y.; Arenal, R.; Benito, A.M.; Maser, W.K.; D’Souza, F.; Tagmatarchis, N. Interfacing Transition Metal Dichalcogenides with Carbon Dots for Managing Photoinduced Energy and Charge-Transfer Processes. J. Am. Chem. Soc. 2018, 140, 13488-13496. [CrossRef]

28. Canton-Vitoria, R.; Sayed-Ahmad-Baraza, Y.; Pelaez-Fernandez, M.; Arenal, R.; Bittencourt, C.; Ewels, C.P.; Tagmatarchis, N. Functionalization of $\mathrm{MoS}_{2}$ with 1,2-Dithiolanes: Toward Donor-Acceptor Nanohybrids for Energy Conversion. npj 2D Mater. Appl. 2017, 1, 13. [CrossRef]

29. Canton-Vitoria, R.; Gobeze, H.B.; Blas-Ferrando, V.M.; Ortiz, J.; Jang, Y.; Fernandez-Lazaro, F.; Sastre-Santos, A.; Nakanishi, Y.; Shinohara, H.; d'Souza, F.; et al. Excited State Charge Transfer in Covalently Functionalized $\mathrm{MoS}_{2}$ with Zinc Phthalocyanine Donor-Acceptor Hybrid. Angew. Chem. Int. Ed. 2019, 58, 5712-5717. [CrossRef]

30. Aleksandrov, L.; Komatsu, T.; Iordanova, R.; Dimitriev, Y. Study of Molybdenum Coordination State and Crystallization Behavior in $\mathrm{MoO}_{3}-\mathrm{La}_{2} \mathrm{O}_{3}-\mathrm{B}_{2} \mathrm{O}_{3}$ Glasses by Raman Spectroscopy. J. Phys. Chem. Solids 2011, 72, 263-268. [CrossRef]

31. Windom, B.C.; Sawyer, W.G.; Hahn, D.W. A Raman Spectroscopic Study of $\mathrm{MoS}_{2}$ and $\mathrm{MoO}_{3}$ : Applications to Tribological Systems. Tribol. Lett. 2011, 42, 301-310. [CrossRef]

32. Daniel, M.F.; Desbat, B.; Lassegues, J.C. Infrared and Raman Study of $\mathrm{WO}_{3}$ Tungsten Trioxides and $\mathrm{WO}_{3}$, $\mathrm{xH}_{2} \mathrm{O}$ Tungsten Trioxide Hydrates. J. Solid State Chem. 1987, 67, 235-247. [CrossRef]

33. Ayala, P.; Arenal, R.; Rummeli, M.; Rubio, A.; Pichler, T. The Doping of Carbon Nanotubes with Nitrogen and Their Potential Applications. Carbon 2010, 48, 575-586. [CrossRef] 
34. Arenal, R.; Blase, X.; Loiseau, A. Boron-Nitride and Boron-Carbonitride Nanotubes: Synthesis, Characterization and Theory. Adv. Phys. 2010,59, 101-179. [CrossRef]

35. Alvarez, L.; Almadori, Y.; Arenal, R.; Babaa, R.; Michel, T.; Leparc, R.; Bantignies, J.-L.; Hermet, P.; Sauvajol, J.-L. Charge Transfer Evidence Between Carbon Nanotubes and Encapsulated Conjugated Oligomers. J. Phys. Chem. C 2011, 115, 11898-11905. [CrossRef]

36. Arenal, R.; De Matteis, L.; Custardoy, L.; Mayoral, A.; Tence, M.; Grazu, V.; de la Fuente, J.M.; Marquina, C.; Ibarra, M.R. Spatially-Resolved EELS Analysis of Antibody Distribution on Biofunctionalized Magnetic Nanoparticles. ACS Nano 2013, 7, 4006-4013. [CrossRef]

37. Albiter, M.A.; Huirache-Acuña, R.; Paraguay-Delgado, F.; Rico, J.L.; Alonso-Nuñez, G. Synthesis of MoS 2 Nanorods and Their Catalytic Test in the HDS of Dibenzothiophene. Nanotechnology 2006, 17, 3473-3481. [CrossRef]

38. Santosh, K.C.; Longo, R.C.; Wallace, R.M.; Cho, K. Surface Oxidation Energetics and Kinetics on $\mathrm{MoS}_{2}$ Monolayer. J. Appl. Phys. 2015, 117, 135301-135309.

39. Addou, R.; McDonnell, S.; Barrera, D.; Guo, Z.; Azcat, A.; Wang, J.; Zhu, H.; Hinkle, C.L.; Quevedo-Lopez, M.; Alshareef, H.N.; et al. Impurities and Electronic Property Variations of Natural $\mathrm{MoS}_{2}$ Crystal Surfaces. ACS Nano 2015, 9, 9124-9133. [CrossRef]

40. Addou, R.; Colombo, L.; Wallace, R.M. Surface Defects on Natural MoS 2 . ACS Appl. Mater. Interfaces 2015, 7, 11921-11929. [CrossRef]

41. Chow, P.; Singh, E.; Viana, B.; Gao, J.; Luo, J.; Li, J.; Lin, Z.; Elías, A.; Shi, Y.; Wang, Z.; et al. Wetting of Mono and Few-Layered $\mathrm{WS}_{2}$ and $\mathrm{Mos}_{2}$ Films Supported on $\mathrm{Si} / \mathrm{SiO}_{2}$ Substrates. ACS Nano 2015, 9, 3023-3031. [CrossRef] [PubMed]

42. Afanasiev, P.; Lorentz, C. Oxidation of Nanodispersed $\mathrm{MoS}_{2}$ in Ambient Air: The Products and the Mechanistic Steps. J. Phys. Chem. C 2019, 123, 7486-7494. [CrossRef]

43. Kozbial, A.; Gong, X.; Liu, H.; Li, L. Understanding the Intrinsic Water Wettability of Molybdenum Disulfide $\left(\mathrm{MoS}_{2}\right)$. Langmuir 2015, 31, 8429-8435. [CrossRef] [PubMed]

44. Barja, S.; Refaely-Abramson, S.; Schuler, B.; Qiu, D.; Pulkin, A.; Wickenburg, S.; Ryu, H.; Ugeda, M.; Kastl, C.; Chen, C. Identifying substitutional oxygen as a prolific point defect in monolayer transition metal dichalcogenides. Nat. Commun. 2019, 10, 3382. [CrossRef]

45. Hu, Z.; Avila, J.; Wang, X.; Leong, J.; Zhang, Q.; Liu, Y.; Asensio, M.; Lu, J.; Carvalho, A.; Sow, C. The Role of Oxygen Atoms on Excitons at the Edges of Monolayer $\mathrm{WS}_{2}$. Nano Lett. 2019, 19, 4641. [CrossRef]

46. Canton-Vitoria, R.; Stangel, C.; Tagmatarchis, N. Electrostatic Association of Ammonium-Functionalized Layered-Transition-Metal Dichalcogenides with an Anionic Porphyrin. ACS Appl. Mater. Interfaces 2018, 10, 23476-23480. [CrossRef]

47. Canton-Vitoria, R.; Vallan, L.; Urriolabeitia, E.; Benito, A.M.; Maser, W.K.; Tagmatarchis, N. Electronic Interactions in Illuminated Carbon Dot/MoS 2 Ensembles and Electrocatalytic Activity Towards Hydrogen Evolution. Chem. Eur. J. 2018, 24, 10468-10474. [CrossRef]

48. Bertolazzi, S.; Gobbi, M.; Zhao, Y.; Backes, C.; Samorì, P. Molecular Chemistry Approaches for Tuning the Properties of Two-Dimensional Transition Metal Dichalcogenides. Chem. Soc. Rev. 2018, 47, 6845-6888. [CrossRef]

49. Stergiou, A.; Tagmatarchis, N. Molecular Functionalization of 2D MoS 2 Nanosheets. Chem. Eur. J. 2018, 24, 18246-18257. [CrossRef]

50. Canton-Vitoria, R.; Istif, E.; Hernandez-Ferrer, J.; Benito, A.M.; Maser, W.K.; Tagmatarchis, N. Integrating Water-Soluble Polythiophene with Transition Metal Dichalcogenides for Managing Photoinduced Processes. ACS Appl. Mater. Interfaces 2019, 11, 5947-5956. [CrossRef]

51. Mirabelli, G.; McGeough, C.; Schmidt, M.; McCarthy, E.; Monaghan, S.; Povey, I.; McCarthy, M.; Gity, F.; Nagle, R.; Hughes, G.; et al. Air Sensitivity of $\mathrm{MoS}_{2}, \mathrm{MoSe}_{2}, \mathrm{MoTe}_{2}, \mathrm{HfS}_{2}$, and HfSe 2 . J. Appl. Phys. 2016, 120, 125102-125111. [CrossRef]

(C) 2020 by the authors. Licensee MDPI, Basel, Switzerland. This article is an open access article distributed under the terms and conditions of the Creative Commons Attribution (CC BY) license (http://creativecommons.org/licenses/by/4.0/). 